\title{
New constitutive equations derived from a kinetic model for melts and concentrated solutions of linear polymers
}

\author{
Jiannong Fang ${ }^{1}$, Robert G. Owens ${ }^{2 \star}$ \\ 1 Ecole Polytechnique Fédérale de Lausanne, Laboratory of Engineering and Environmental Geology, \\ CH-1015 Lausanne, Switzerland. \\ 2 Ecole Polytechnique Fédérale de Lausanne, Fluid Mechanics Laboratory, \\ CH-1015 Lausanne, Switzerland.
}

Received: date / Revised version: date

\begin{abstract}
In this paper new constitutive equations for linear entangled polymer solutions and melts are derived from a recently proposed kinetic model (Fang et al. 2004) by using five closure approximations available in the literature. The simplest closure approximation considered is that due to Peterlin (1966). In this case a mean-fieldtype Fokker-Planck equation underlying the evolution equation for an equilibrium averaged polymer segment orientation tensor is shown to be consistent with the fluctuation-dissipation theorem (Kubo et al. 1985). We compare the performance of the five new constitutive equations in their capacity to faithfully reproduce the predictions of the modified encapsulated FENE dumbbell model of Fang et al. (2004) for a number of shear and extensional flows. Comparisons are also made with the experimental data of Kahvand (1995) and Bhattacharjee et al. $(2002,2003)$. In the case of the Hinch-Leal and Bingham closures (Hinch and Leal 1976; Chaubal and Leal 1998) a combination with the quadratic closure of Doi (1981) is found to be necessary for stability in fast flows. The Hinch-Leal closure approximation, modified in this way, is found to outperform the other closures and its mathematical description is considerably simpler than that of the Bingham closure.
\end{abstract}

Key words Linear entangled polymers - closure approximations - encapsulated FENE dumbbell - reptation - constitutive equations

\section{Introduction}

The irreversible trend in present day constitutive modelling and computation of flows of complex (non-Newtonian)

Send offprint requests to: jiannong.fang@epfl.ch

* Present address: Département de mathématiques et de statistique, Université de Montréal, CP 6128 succ. CentreVille, Montréal QC H3C 3J7, Canada. fluids is towards so-called "micro-macro" methods where the numerical solution to the macroscopic equations of conservation of mass and linear momentum are coupled with the determination of an elastic stress from a kinetic theory model. A concern for fidelity in the modelling of what may often be very complex rheological behaviour generally precludes the use of simple approximations using closed-form constitutive equations and may necessitate a stochastic approach or, in the case of concentrated polymer solutions and melts, a reptationtype model (Doi and Edwards 1978a,b,c; Mead et al. 1998; Öttinger 1999). The effective modelling of flows of entangled polymers is a particularly challenging task and modern reptation theory incorporates such nonlinear properties as chain stretching (Marrucci and Grizzuti 1988; Mead and Leal 1995; Mead et al. 1995; Pearson et al. 1991), double reptation (des Cloizeaux 1988; Öttinger 1994; Tsenoglu 1987), convective constraint release (CCR) (Ianniruberto and Marrucci 1996; Marrucci 1996) and convective conformal renewal (CCR2) due to flow-induced lengthening of tube segments (Ianniruberto and Marrucci 2000, 2001). A short summary of these effects may be found in the paper of Fang et al. (2000) and a review of some current coarse-grained reptation models is given by Fang et al. (2004).

Very recently, Fang et al. (2004) have proposed a single segment stochastic model for flows of concentrated solutions of linear polymers which is a modification of the original encapsulated FENE dumbbell (EFD) model of Bird and Deaguiar (1983). The micro-mechanical model to which the original EFD model corresponds is that of a polymer molecule represented by a dumbbell consisting of two beads having mass $m$ joined by a massless spring and subject, as the dumbbell moves in a Newtonian solvent, to an anisotropic friction force due to the presence of other molecules. The two beads at either end of the 
dumbbell satisfy the equations of motion

$$
\begin{aligned}
m d\left(\frac{d \boldsymbol{r}_{i}}{d t}-\boldsymbol{v}\left(\boldsymbol{r}_{i}\right)\right)=- & \boldsymbol{\zeta} \\
& \left(\frac{d \boldsymbol{r}_{i}}{d t}-\boldsymbol{v}\left(\boldsymbol{r}_{i}\right)\right) d t+\boldsymbol{F}_{i}^{(c)} d t \\
& +\sqrt{2 k_{B} T \boldsymbol{\zeta}} \cdot d \boldsymbol{W}_{i}, \quad(i=1,2)
\end{aligned}
$$

where $\boldsymbol{r}_{i}$ is the position vector of the $i$ th bead, $\boldsymbol{v}\left(\boldsymbol{r}_{i}\right)$ is the velocity of the solvent at the point with position vector $\boldsymbol{r}_{i}$ and $\boldsymbol{F}_{i}^{(c)}$ is the entropic FENE spring force acting on the $i$ th bead. Let $\boldsymbol{Q}=\boldsymbol{r}_{2}-\boldsymbol{r}_{1}$ denote the end-to-end vector of the dumbbell so that $\boldsymbol{u}=\boldsymbol{Q} / Q$ is the unit direction vector. Then in the EFD model $\boldsymbol{\zeta}$ is an anisotropic friction tensor that may be written in the form

$$
\boldsymbol{\zeta}=\zeta\left(\boldsymbol{u} \boldsymbol{u}+\sigma^{-1}(\boldsymbol{\delta}-\boldsymbol{u} \boldsymbol{u})\right)
$$

and in this expression $\zeta$ is a friction coefficient and $\sigma \leq 1$ a parameter determining the extent of frictional anisotropy. The final term in Eq. (1) is a stochastic force due to the bombardment of the beads by the surrounding solvent molecules and determined by the fluctuation-dissipation theorem (see, for example, Section 2 of Schieber 1992), where $\boldsymbol{W}_{i}$ denotes a multi-dimensional Wiener process.

Introducing

$$
\boldsymbol{F}^{(c)}:=\boldsymbol{F}_{1}^{(c)}\left(=-\boldsymbol{F}_{2}^{(c)}\right),
$$

the FENE spring force law may be written as

$$
\boldsymbol{F}^{(c)}=\frac{H \boldsymbol{Q}}{1-Q^{2} / Q_{\max }^{2}},
$$

where $Q_{\max }$ is the maximum extensibility of the dumbbell spring. With $\zeta$ and $H$ as given in the definitions of the anisotropic friction tensor $\boldsymbol{\zeta}$ and spring force law $\boldsymbol{F}^{(c)}$ the time constants $\lambda_{H}=\zeta /(4 H)$ and $\lambda_{B}=m / \zeta$ may be interpreted as relaxation times for the dumbbell configuration and for the dumbbell velocity, respectively. By taking the limit of zero dimensionless mass $\lambda_{B} / \lambda_{H}$ it may then be shown (see Schieber and Öttinger 1998, for example) that the stochastic equation for the EFD model may be written in dimensionless form as

$$
\begin{gathered}
d \boldsymbol{Q}=\left\{\boldsymbol{\kappa} \cdot \boldsymbol{Q}-\frac{1}{\tau_{s}} \frac{\boldsymbol{Q}}{\left(1-Q^{2} / b\right)}+\left(\frac{2}{\tau_{s}}-2 D\right) \frac{\boldsymbol{u}}{Q}\right\} d t \\
+\left\{\sqrt{\frac{2}{\tau_{s}}} \boldsymbol{u} \boldsymbol{u}+\sqrt{2 D}(\boldsymbol{\delta}-\boldsymbol{u} \boldsymbol{u})\right\} \cdot d \boldsymbol{W}_{t}
\end{gathered}
$$

where we have assumed that the flow is homogeneous so that there exists a constant transposed velocity gradient $\boldsymbol{\kappa}$ (say) such that $\boldsymbol{v}\left(\boldsymbol{r}_{i}\right)=\boldsymbol{v}(\mathbf{0})+\boldsymbol{\kappa} \cdot \boldsymbol{r}_{i}$. In Eq. (5) $\tau_{s}:=$ $2 \lambda_{H}, D:=\sigma /\left(2 \lambda_{H}\right), \boldsymbol{Q}$ has been non-dimensionalized by scaling it with $\sqrt{k_{B} T / H}, b$ is a dimensionless maximum spring extensibility and $\boldsymbol{W}_{t}:=\left(\boldsymbol{W}_{2}-\boldsymbol{W}_{1}\right) / \sqrt{2}$.

In the modification to the original EFD model of Bird and Deaguiar (1983) proposed by Fang et al. (2004) $\boldsymbol{Q}$ was interpreted as the end-to-end vector of an entanglement segment in a bead-spring chain and the orientation diffusion coefficient $D$ redefined as $D=1 /\left(6 \tau_{\text {eff }}\right)$ with the following formulation of the effective orientation relaxation time $\tau_{\text {eff }}$ :

$$
\tau_{\text {eff }}=\max \left(\frac{1}{\frac{2}{\tau_{d}}+\beta_{1} k H(k)}, \tau_{R}\right) .
$$

In (6) $H=H(k)$ is the Heaviside step function and the constraint release rate $k$ is defined by

$$
k=\boldsymbol{\kappa}:\langle\boldsymbol{u} \boldsymbol{u}\rangle-\frac{\langle\dot{Q}\rangle}{\langle Q\rangle},
$$

where, denoting by $\psi$ the configuration probability density function, $\langle\cdot\rangle$ here and henceforth in this paper denotes the configuration space average

$$
\langle\cdot\rangle=\int_{\boldsymbol{Q}} \cdot \psi(\boldsymbol{Q}, t) d \boldsymbol{Q} .
$$

Fang et al. (2004) described how the definition of the diffusion coefficient $D$ in terms of $\tau_{\text {eff }}$ above accounts for double reptation (through the reptation time $\tau_{d}$ ) and convective constraint release (CCR) through $k$. The factor $\beta_{1}$ appearing in Eq. (6) allows convective conformation renewal (CCR2) due to flow-induced lengthening of tube segments (Ianniruberto and Marrucci 2000, 2001) and $\beta_{1} k H(k)$ determines the effective orientation relaxation rate caused by constraint releases. The term involving $\tau_{R}$ (the so-called Rouse time) is an irreducible friction term whose presence indicates the fact that once the topological contribution to the chain friction is swept away the orientational relaxation time of the now unentangled chain drops to the Rouse time (see the papers of Ianniruberto and Marrucci 2001, 2002).

For the characteristic stretching relaxation time $\tau_{s}$ in (5), the alternative form proposed by Fang et al. (2004) was

$$
\tau_{s}=\max \left(\frac{1}{\frac{1}{\tau_{R 0}}+\beta_{2} k H(k)}, \tau_{R}\right),
$$

where $\tau_{R 0}$ is the primary Rouse time at equilibrium. The argument used by the authors to justify the choice of (8) was that $\tau_{s}$ should also vary with CCR from the value at equilibrium under constraint to the value corresponding to a somehow unconstrained Rouse chain, because of the fast removal of constraints. Denoting the number of entanglements in the model polymer by $Z$, Fang et al. (2004) chose $\tau_{d} / \tau_{R}=3 Z$ and $\tau_{d} / \tau_{R 0}=Z$, consistent with the fact that at equilibrium (entangled case) stretch relaxation is simply along the contour path of the chain and thus essentially one-dimensional, whereas the assumption underlying the choice of the pre-factor 3 in the choice of $3 Z$ for the reptation to Rouse time ratio in the original paper of Doi and Edwards was that relaxation occurred in three dimensions (Meerveld 2002). Stretch relaxation therefore takes place over a longer time under equilibrium conditions than in a fast flow, as seems intuitively reasonable. Despite this, it has been 
usual within the scientific literature (see, for example, Fang et al. 2000) to choose a fixed ratio $\tau_{d} / \tau_{s}=3 Z$ for the characteristic reptation and stretching times.

Fang et al. (2004) demonstrated that agreement in the predictions of their model with the experimental results of Bhattacharjee et al. (2002, 2003) for steady shear flow and uniaxial extensional flow of a $10 \%$ solution of polystyrene in diethyl phthalate and with the experimental observations of Venerus and Kahvand (1994) for reversing double-step strain flow of a $12 \mathrm{wt} \%$ solution of polystyrene in tricresyl phosphate were excellent and better than those obtained with the recent coupled DCR-CS model of Marrucci and Ianniruberto (2003). The great advantage that the latter model has over that of Fang et al. (2004), however, is that being in the form of a comparatively simple deterministic differential equation for the second moment of the segment end-to-end vector of a polymer segment, computation of the stress is much cheaper than via a stochastic description. The motivation of this paper, then, is the search, necessarily involving a compromise between complexity and accuracy, for a deterministic differential equation offering on the one hand a very cheap alternative to the stochastic differential equation (5), yet on the other close agreement with the predictions of the model of Fang et al. (2004) for (at least) simple shear and extensional flows. One possibility, of course, is to write down and solve the Fokker-Planck equation equivalent to Eq. (5). Several recent studies (Lozinski and Chauvière 2003; Lozinski et al. 2003; Chauvière and Lozinski 2004; Lozinski et al. 2004) have shown that for problems involving weak flows and low-dimensional configuration spaces, FokkerPlanck-based numerical methods offer an attractive alternative to stochastic techniques, at a much reduced $\mathrm{CPU}$ cost. Although the probability density function in the Fokker-Planck equation equivalent to Eq. (5) is only a function of time $(t)$ and $\boldsymbol{Q}$ (a three-dimensional variable) and therefore tractable using Fokker-Planck-based numerical methods, this Fokker-Planck equation would in general still be much more expensive to solve than, for example, a set of differential equations valid throughout the flow domain. Naturally, we turn our attention, therefore, to closure approximations of the model of Fang et al. (2004). The construction of closure approximations may occasionally seem more reminiscent of black art than science although some physical guiding principles may be found in the literature (see, for example, the paper by Zmievski et al. (2000)). Certainly, the use of low-dimensional canonical distribution functions, for example, would appear to be on a solid footing (see further comments in Section 3). For more details we refer the reader to several discussions of the construction of closure approximations that have appeared in the literature over the past forty years or so (Hand 1962; Hinch and Leal 1975, 1976; Advani and Tucker 1987; Maffettone 1992; Cintra and Tucker 1995; Chaubal and Leal 1998; Feng et al. 1998; Grosso et al. 2000a, 2000b).
In the next section of the present paper we adapt five different closures (Peterlin 1966; Lielens et al. 1999; Doi 1981; Hinch and Leal 1976; Chaubal and Leal 1998) to the approximation of the Fang et al. (2004) model. In the section that follows afterwards we consider the validity of the fluctuation-dissipation theorem (Kubo et al. 1985) for the Peterlin approximation and then study the agreement of the closure approximations in a number of shear and extensional flows with the predictions of the modified EFD model and with the experimental data of Bhattacharjee et al. $(2002,2003)$ and Kahvand (1995). Finally, we draw some conclusions and make a recommendation based upon our observations.

\section{The closure models}

To derive closed-form constitutive equations from the kinetic model of Fang et al. (2004) described in the Introduction, we consider the closure problem for the kinetic theory of polymer solutions based upon FENE dumbbells.

As a starting point, let us define a state variable $X$ which is the configuration space average of a scalar or tensorial function $f$ of the configurational variable $Q$ :

$$
X=\langle f(\boldsymbol{Q})\rangle .
$$

It may then be shown (see Bird et al. 1987, for example) that $X$ satisfies the following time evolution equation:

$$
\begin{aligned}
\frac{D X}{D t} & =\left\langle\frac{\partial}{\partial \boldsymbol{Q}} \cdot\left[\frac{1}{\tau_{s}} \boldsymbol{u u}+D(\boldsymbol{\delta}-\boldsymbol{u u})\right] \cdot \frac{\partial}{\partial \boldsymbol{Q}} f(\boldsymbol{Q})\right\rangle \\
& +\boldsymbol{\kappa}:\left\langle\boldsymbol{Q} \frac{\partial}{\partial \boldsymbol{Q}} f(\boldsymbol{Q})\right\rangle-\frac{1}{\tau_{s}}\left\langle\boldsymbol{F}^{c}(\boldsymbol{Q}) \cdot \frac{\partial}{\partial \boldsymbol{Q}} f(\boldsymbol{Q})\right\rangle .
\end{aligned}
$$

If, therefore, we choose as the state variable $X=X_{1}$, where $X_{1}$ is the second moment of the end-to-end vector

$$
X_{1}=\boldsymbol{A}=\langle Q Q\rangle
$$

the evolution equation (10) for $X_{1}$ reads

$$
\begin{array}{r}
\frac{D \boldsymbol{A}}{D t}=2 D \boldsymbol{\delta}+\boldsymbol{\kappa} \cdot \boldsymbol{A}+\boldsymbol{A} \cdot \boldsymbol{\kappa}^{T}-\frac{2}{\tau_{s}}\left\langle\boldsymbol{Q} \boldsymbol{F}^{c}(\boldsymbol{Q})\right\rangle \\
+6\left(\frac{1}{\tau_{s}}-D\right)\langle\boldsymbol{u} \boldsymbol{u}\rangle .
\end{array}
$$

Clearly, the two new state variables $\boldsymbol{T}=\left\langle\boldsymbol{Q} \boldsymbol{F}^{c}(\boldsymbol{Q})\right\rangle$ and $\boldsymbol{S}=\langle\boldsymbol{u} \boldsymbol{u}\rangle$ (an orientation tensor) in Eq. (12) introduce a closure problem. Once $\boldsymbol{T}$ has been determined the polymeric stress $\tau$ may be calculated from

$$
\boldsymbol{\tau}=G \boldsymbol{T},
$$

where $G=\nu k_{B} T$ is an elastic modulus and $\nu$ denotes the segment density. Of the very many closure approximations that are available in the literature we review five below. All of these lead to systems of differential 
equations of varying complexity from which $\boldsymbol{T}$ may be found.

Three of the closure approximations in our review (the Peterlin, FENE-LS and Bingham closures) may be derived by approximating $\psi$ with a function $\psi^{c}$ (say) drawn from a low-dimensional canonical distribution. In the case of the Peterlin and FENE-LS closure approximations the canonical distribution function $\psi^{c}$ is assumed to be length and orientation separable. That is, we may write

$$
\psi^{c}(\boldsymbol{Q})=\psi^{Q}(Q) \psi^{u}(\boldsymbol{u})
$$

with the following normalization:

$$
\psi^{u}(\boldsymbol{u}) \geq 0, \oint \psi^{u}(\boldsymbol{u}) d \boldsymbol{u}=1
$$

and

$$
\psi^{Q}(Q) \geq 0, \int_{0}^{\sqrt{b}} Q^{2} \psi^{Q}(Q) d Q=1
$$

Let $\langle\cdot\rangle_{c}$ denote the configuration space average computed with the canonical distribution function $\psi^{c}$. Then

$$
\begin{aligned}
\boldsymbol{A} \approx\langle\boldsymbol{Q} \boldsymbol{Q}\rangle_{c} & =\int_{\boldsymbol{Q}} \boldsymbol{Q} \boldsymbol{Q} \psi^{c}(\boldsymbol{Q}) d \boldsymbol{Q} \\
& =\int_{0}^{\sqrt{b}} Q^{2} \rho^{c}(Q) d Q \oint \boldsymbol{u} \boldsymbol{u} \psi^{u}(\boldsymbol{u}) d \boldsymbol{u}
\end{aligned}
$$

Since $\boldsymbol{u}$ is a unit vector and using (15) it follows from taking the trace throughout (17) that

$$
\int_{0}^{\sqrt{b}} Q^{2} \rho^{c}(Q) d Q \approx \operatorname{tr}(\boldsymbol{A})
$$

and hence that

$$
\oint \boldsymbol{u} u \psi^{u}(\boldsymbol{u}) d \boldsymbol{u} \approx \frac{\boldsymbol{A}}{\operatorname{tr}(\boldsymbol{A})} .
$$

From (16) and (19) we thus get

$$
\boldsymbol{S} \approx\langle\boldsymbol{u} \boldsymbol{u}\rangle_{c}=\int_{0}^{\sqrt{b}} Q^{2} \psi^{Q} d Q \oint \boldsymbol{u} \boldsymbol{u} \psi^{u}(\boldsymbol{u}) d \boldsymbol{u}=\frac{\boldsymbol{A}}{\operatorname{tr}(\boldsymbol{A})}
$$

The Peterlin and FENE-LS closure approximations differ in the choice of the normalized radial distribution $\rho^{c}(Q)=Q^{2} \psi^{Q}(Q)$.

\subsection{The Peterlin closure}

The classical Peterlin closure approximation (Peterlin 1966) amounts to choosing the single parameter canonical distribution

$$
\rho_{\alpha}^{c}=\delta(Q-\alpha)
$$

where $\delta$ is the delta function and the parameter $\alpha \in$ $(0, \sqrt{b})$. From $(18)$ we then get $\operatorname{tr}(\boldsymbol{A}) \approx \alpha^{2}$. Thus, writing

$$
\boldsymbol{T} \approx\left\langle\boldsymbol{Q F}^{c}(\boldsymbol{Q})\right\rangle_{c}=\int_{\boldsymbol{Q}} \frac{\boldsymbol{Q} \boldsymbol{Q}}{1-Q^{2} / b} \psi^{c}(\boldsymbol{Q}) d \boldsymbol{Q},
$$

it may be seen that

$$
\begin{aligned}
\boldsymbol{T} & \approx \int_{0}^{\sqrt{b}} \frac{Q^{2}}{1-Q^{2} / b} \rho_{\alpha}^{c}(Q) d Q \oint \boldsymbol{u} \boldsymbol{u} \psi^{u}(\boldsymbol{u}) d \boldsymbol{u} \\
& =\frac{\alpha^{2}}{1-\alpha^{2} / b} \oint \boldsymbol{u} \boldsymbol{u} \psi^{u}(\boldsymbol{u}) d \boldsymbol{u}=\frac{\boldsymbol{A}}{1-\operatorname{tr}(\boldsymbol{A}) / b} .
\end{aligned}
$$

From (12) we obtain what we will henceforth term the MEFD-P model:

$$
\begin{aligned}
\frac{D \boldsymbol{A}}{D t}=2 D \boldsymbol{\delta}+\boldsymbol{\kappa} \cdot \boldsymbol{A}+\boldsymbol{A} \cdot \boldsymbol{\kappa}^{T} & -\frac{2}{\tau_{s}} \frac{\boldsymbol{A}}{1-\operatorname{tr}(\boldsymbol{A}) / b} \\
+ & 6\left(\frac{1}{\tau_{s}}-D\right) \frac{\boldsymbol{A}}{\operatorname{tr}(\boldsymbol{A})} .
\end{aligned}
$$

Accordingly, the constraint release rate $k$ and the stress expression (13) are approximated as

$$
k=\frac{\boldsymbol{\kappa}: \boldsymbol{A}}{\operatorname{tr}(\boldsymbol{A})}-\frac{1}{\sqrt{\operatorname{tr}(\boldsymbol{A})}} \frac{d}{d t} \sqrt{\operatorname{tr}(\boldsymbol{A})}
$$

and

$$
\boldsymbol{\tau}=\nu k_{B} T \boldsymbol{T}=\nu k_{B} T \frac{\boldsymbol{A}}{1-\operatorname{tr}(\boldsymbol{A}) / b},
$$

respectively.

\subsection{The FENE-LS closure}

We now wish to develop a second-order closure by introducing a second state variable $X_{2}=B=\left\langle Q^{4}\right\rangle$. The evolution equation (10) for $X_{2}$ reads

$$
\frac{D B}{D t}=\frac{20}{\tau_{s}} \operatorname{tr}(\boldsymbol{A})+4 \boldsymbol{\kappa}:\left\langle Q^{2} \boldsymbol{Q} \boldsymbol{Q}\right\rangle-\frac{4}{\tau_{s}}\left\langle\frac{Q^{4}}{1-Q^{2} / b}\right\rangle .
$$

Here, closure is needed for the two new state variables $\boldsymbol{B}_{1}=\left\langle Q^{2} \boldsymbol{Q} \boldsymbol{Q}\right\rangle$ and $B_{2}=\left\langle Q^{4} /\left(1-Q^{2} / b\right)\right\rangle$ appearing in the above equation. With $\langle\cdot\rangle_{c}$ denoting, as before, the configuration space average computed with the canonical distribution function $\psi^{c}$, it follows that

$$
B \approx\left\langle Q^{4}\right\rangle_{c}=\int_{0}^{\sqrt{b}} Q^{4} \rho^{c}(Q) d Q,
$$

$$
\begin{gathered}
\boldsymbol{T} \approx\left\langle\boldsymbol{Q} \boldsymbol{F}^{c}(\boldsymbol{Q})\right\rangle_{c}=\frac{\boldsymbol{A}}{\operatorname{tr}(\boldsymbol{A})} \int_{0}^{\sqrt{b}} \frac{Q^{2}}{1-Q^{2} / b} \rho^{c}(Q) d Q \\
\boldsymbol{S} \approx\langle\boldsymbol{u} \boldsymbol{u}\rangle_{c}=\frac{\boldsymbol{A}}{\operatorname{tr}(\boldsymbol{A})}, \\
\boldsymbol{B}_{1} \approx\left\langle Q^{2} \boldsymbol{Q} \boldsymbol{Q}\right\rangle_{c}=\frac{\boldsymbol{A}}{\operatorname{tr}(\boldsymbol{A})} B,
\end{gathered}
$$


and

$$
B_{2} \approx\left\langle Q^{4} /\left(1-Q^{2} / b\right)\right\rangle_{c}=\int_{0}^{\sqrt{b}} \frac{Q^{4}}{1-Q^{2} / b} \rho^{c}(Q) d Q .
$$

Now only the scalar state variables

$$
A^{C}=\int_{0}^{\sqrt{b}} \frac{Q^{2}}{1-Q^{2} / b} \rho^{c}(Q) d Q
$$

and

$$
B^{C}=\int_{0}^{\sqrt{b}} \frac{Q^{4}}{1-Q^{2} / b} \rho^{c}(Q) d Q
$$

need closure. The two-parameter canonical FENE-LS radial distribution introduced by Lielens et al. (1999) was

$$
\rho_{\alpha, \beta}^{c}=(1-\beta) \delta(Q-\alpha / R)+\beta \delta(Q-\alpha),
$$

where $(\alpha, \beta) \in(0, \sqrt{b}) \times[0,1]$ and $R$ is a constant. Using this distribution,

$$
\begin{gathered}
\operatorname{tr}(\boldsymbol{A}) \approx \alpha^{2}\left(\beta+\frac{(1-\beta)}{R^{2}}\right), \\
B \approx \alpha^{4}\left(\beta+\frac{(1-\beta)}{R^{4}}\right), \\
A^{C} \approx \alpha^{2}\left(\beta \frac{1}{1-\alpha^{2} / b}+\frac{(1-\beta)}{R^{2}} \frac{1}{1-\alpha^{2} /\left(R^{2} b\right)}\right), \\
B^{C} \approx \alpha^{4}\left(\beta \frac{1}{1-\alpha^{2} / b}+\frac{(1-\beta)}{R^{4}} \frac{1}{1-\alpha^{2} /\left(R^{2} b\right)}\right) .
\end{gathered}
$$

The relations (36) and (37) yield $\alpha$ and $\beta$ as a function of $A=\operatorname{tr}(\boldsymbol{A})$ and $d=B / A^{2}$ :

$$
\begin{aligned}
\alpha^{2} & =\frac{2 A R^{2} d}{\left(R^{2}+1\right)+\left(R^{2}+1\right) \sqrt{1-\left[4 R^{2} d /\left(R^{2}+1\right)^{2}\right]}}, \\
\beta & =\frac{R^{2}\left(A / \alpha^{2}\right)-1}{R^{2}-1} .
\end{aligned}
$$

Inserting the above results into (38) and (39) gives the FENE-LS closure. For calculating the constraint release rate $k,\langle Q\rangle$ can be approximated as

$$
\langle Q\rangle \approx\langle Q\rangle_{c}=\int_{0}^{\sqrt{b}} Q \rho_{\alpha, \beta}^{c}(Q) d Q=\alpha(\beta+(1-\beta) / R) .
$$

The model consisting of the evolution equations (12) and (27) together with the above closure relations will be called MEFD-LS in the sequel.

We would now like to obtain the orientation tensor $\boldsymbol{S}=\langle\boldsymbol{u} \boldsymbol{u}\rangle$ in what may be hoped to be a more accurate way than via the approximation (20). To this end we derive the following evolution equation for $\boldsymbol{S}$ from (10):

$$
\begin{array}{r}
\frac{D S}{D t}=2 D \boldsymbol{\delta}\left\langle\frac{1}{Q^{2}}\right\rangle+\boldsymbol{\kappa} \cdot \boldsymbol{A}+\boldsymbol{A} \cdot \boldsymbol{\kappa}^{T}-6 D\left\langle\frac{\boldsymbol{u u}}{Q^{2}}\right\rangle \\
-2 \boldsymbol{\kappa}:\langle\boldsymbol{u u u \boldsymbol { u } \rangle} .
\end{array}
$$

Here

$$
S_{1}=\left\langle\frac{1}{Q^{2}}\right\rangle,
$$

$$
\boldsymbol{S}_{2}=\left\langle\frac{u u}{Q^{2}}\right\rangle
$$

and

$$
\boldsymbol{R}=\langle\boldsymbol{u} u \boldsymbol{u u}\rangle,
$$

need to be closed. For closing $S_{1}$ and $\boldsymbol{S}_{\mathbf{2}}$, either the Peterlin or the FENE-LS closure can be used. By applying the Peterlin closure, we have

$$
S_{1} \approx \frac{1}{\operatorname{tr}(\boldsymbol{A})}, \quad \boldsymbol{S}_{2} \approx \frac{\boldsymbol{S}}{\operatorname{tr}(\boldsymbol{A})}
$$

By applying the FENE-LS closure, we have

$$
S_{1} \approx \frac{\beta+R^{2}(1-\beta)}{\alpha^{2}}, \quad \boldsymbol{S}_{2} \approx S_{1} \boldsymbol{S} .
$$

Let us consider three different closures for $\boldsymbol{R}$ : the quadratic closure of Doi (1981), a closure relation due to Hinch and Leal (1976) and another by Chaubal and Leal (1998), based upon the set of Bingham distributions.

\subsection{The quadratic closure}

The simplest way to close $\boldsymbol{R}$ is using the quadratic closure originally adopted by Doi (1981) for the modelling of liquid-crystalline polymers:

$$
\langle\boldsymbol{u u u u}\rangle=\langle\boldsymbol{u u}\rangle\langle\boldsymbol{u u}\rangle=\boldsymbol{S S} .
$$

As noted by Feng et al. (1998) in a comparative study of closure models for the simulation of complex flows of liquid-crystalline polymers, however, the quadratic closure (48) has weaknesses, most notably that in simple shear flow the director tumbling and wagging predicted by the exact Doi theory for rigid rod molecules is not reproduced. We note that the quadratic closure is only exact if $\psi(\boldsymbol{Q})$ is a Dirac delta function $\delta(\boldsymbol{Q}-\widehat{\boldsymbol{Q}})$ centred on some direction $\widehat{\boldsymbol{Q}}$.

The model consisting of the equations (12), (42), (46) and (48) will be denoted by MEFD-QP and that consisting of (12), (27), (42), (47) and (48) will be referred to henceforth as MEFD-Q. Note that although $\boldsymbol{S}$ should be used directly in calculating $k$ in Eq. (7), we found that leaving $\langle\boldsymbol{u} \boldsymbol{u}\rangle$ approximated by $\boldsymbol{A} / \operatorname{tr}(\boldsymbol{A})$ gave better results. It is not entirely clear to us why this should be so but a conjecture is put forward in Section 3.3.2. 


\subsection{The Hinch and Leal (HL) closure}

Hinch and Leal (1976) proposed two closures for $\boldsymbol{R}$ in the context of approximate constitutive equations for dilute suspensions of rigid spheroidal particles. The idea behind the construction of the proposed closure approximations was an interpolation between the weak flow (near equilibrium) and strong flow (weak Brownian motion) asymptotic expressions at different orders for the relationship between the averaged quantities $\boldsymbol{R}$ and $\boldsymbol{S}$. A simple linear combination of the strong flow asymptotic expressions at the lowest order, having the correct weak flow behaviour, was supplied by Hinch and Leal:

$$
\boldsymbol{D}:\langle\boldsymbol{u} u \boldsymbol{u} u\rangle=\frac{1}{5}[6 \boldsymbol{S} \cdot \boldsymbol{D} \cdot \boldsymbol{S}-\boldsymbol{D}: \boldsymbol{S} \boldsymbol{S}+2 \boldsymbol{\delta}(\boldsymbol{S}-\boldsymbol{S} \cdot \boldsymbol{S}): \boldsymbol{D}],
$$

where $\boldsymbol{D}$ is any traceless and symmetric tensor. The closure (49) shall be referred to as the HL closure from this point on. In order to apply the HL closure to our case, we first observe that since the fourth order tensor $\boldsymbol{R}$ is symmetric with respect to all its indices and therefore, in particular, with respect to its first two,

$$
\boldsymbol{\kappa}:\langle\boldsymbol{u} \boldsymbol{u} \boldsymbol{u} \boldsymbol{u}\rangle=\frac{1}{2}\left(\boldsymbol{\kappa}+\boldsymbol{\kappa}^{T}\right):\langle\boldsymbol{u u u u}\rangle
$$

The HL closure was shown by Chaubal (1997) to be more accurate than the quadratic closure. However, we found that, when applied to the modified EFD model, it fails to produce a stable steady state for start-up of extensional flow at high extension rates. Hence, noting that $\operatorname{tr}(\boldsymbol{A}) \rightarrow$ $b$ as the extension rate increases, we propose to combine the HL closure and the quadratic closure in the following way:

$$
\begin{aligned}
\boldsymbol{\kappa}:\langle\boldsymbol{u u u u}\rangle= & \frac{1}{2}\left(1-(\operatorname{tr}(\boldsymbol{A}) / b)^{\omega}\right)\left(\boldsymbol{\kappa}+\boldsymbol{\kappa}^{T}\right):\langle\boldsymbol{u u u \boldsymbol { u }}\rangle_{H L} \\
& +(\operatorname{tr}(\boldsymbol{A}) / b)^{\omega} \boldsymbol{\kappa}: \boldsymbol{S} \boldsymbol{S}
\end{aligned}
$$

for some suitable choice of the parameter $\omega(\omega=0.5$ is used for the results presented in Section 3). The model obtained will be called MEFD-HL if the FENE-LS closure is used for the approximation of the $\boldsymbol{T}, S_{1}$ and $\boldsymbol{S}_{\mathbf{2}}$ terms and MEFD-HLP if the Peterlin closure is used for those terms.

\subsection{The Bingham closure}

The Bingham closure adopted in this paper was presented recently by Grosso et al. (2000a, 2000b) and evaluated by these authors in a shear flow and the start-up flow of a nematic rod-like polymer in an eccentric cylinder geometry. The closure approximation for $\boldsymbol{R}$ in terms of $\boldsymbol{S}$ was derived in the framework of a canonical distribution function based upon two parameters and belonging to the set of Bingham functions (see Chaubal and Leal
Table 1 The numerical values of the parameters appearing in Eq. (53)

\begin{tabular}{llll}
\hline$p_{1}$ & -9.6746 & $p_{7}$ & 10.6180 \\
$p_{2}$ & -53.486 & $p_{8}$ & -1.3120 \\
$p_{3}$ & 14.4619 & $p_{9}$ & 14.1101 \\
$p_{4}$ & -2.2786 & $p_{10}$ & -68.022 \\
$p_{5}$ & -9.0389 & $k_{1}$ & 4.5906 \\
$p_{6}$ & 108.098 & $k_{2}$ & -12.3104 \\
\hline
\end{tabular}

1998). That is, a canonical distribution function $\psi^{c}$ of the form

$$
\begin{aligned}
\psi^{c}\left(\boldsymbol{u}, \lambda_{1}, \lambda_{2}\right) & =\frac{1}{Z} \exp \left(\boldsymbol{u}^{T} \boldsymbol{C u}\right) \\
= & \frac{1}{Z} \exp \left(\lambda_{1} \cos ^{2} \varphi \sin ^{2} \theta+\lambda_{2} \sin ^{2} \varphi \sin ^{2} \theta\right. \\
& \left.-\left(\lambda_{1}+\lambda_{2}\right) \cos ^{2} \theta\right)
\end{aligned}
$$

was used, where $Z$ is a normalization constant, $\boldsymbol{C}$ is a real symmetric matrix having eigenvalues $\lambda_{1}, \lambda_{2}$ and $-\left(\lambda_{1}+\lambda_{2}\right)$ and $\boldsymbol{u}$ may be written in terms of the spherical coordinates $\theta$ and $\varphi$ as $\boldsymbol{u}=(\sin \theta \cos \varphi, \sin \theta \sin \varphi, \cos \theta)$.

Following Grosso et al. (2000a, 2000b) the Bingham closure for $\boldsymbol{R}$ may be written as

$$
\begin{aligned}
R_{i j k l}= & c_{1} \overline{\delta_{i j} \delta_{k l}}+c_{2} \overline{\delta_{i j} S_{k l}}+c_{3} \overline{\delta_{i j} S_{k m} S_{m l}}+c_{4} \overline{S_{i j} S_{k l}} \\
& +c_{5} \overline{S_{i j} S_{k m} S_{m l}}+c_{6} \overline{S_{i m} S_{m j} S_{k n} S_{n l}} \\
c_{4}= & k_{1}+p_{1} I_{2}+p_{2} I_{3}+p_{3} I_{2}^{2} \\
c_{5}= & p_{4}+p_{5} I_{2}+p_{6} I_{3}+p_{7} I_{2}^{2} \\
c_{6}= & p_{8}+p_{9} I_{2}+p_{10} I_{3}+k_{2} I_{2}^{2} \\
c_{3}= & \frac{1}{7}\left(-4 c_{4}-5 c_{5}+2\left(4 I_{2}-3\right) c_{6}\right) \\
c_{2}= & \frac{1}{7}\left(\left(6 I_{2}-1\right) c_{5}+4\left(I_{2}-I_{3}\right) c_{6}-2 c_{4}+6\right) \\
c_{1}= & \frac{1}{10}\left(\left(2 I_{2}-1\right) c_{3}-4 I_{3} c_{5}-4 I_{3} c_{6}-c_{2}\right)
\end{aligned}
$$

In the above equations, the overbar implies the operation which makes the tensor fully symmetric, and $I_{2}$ and $I_{3}$ represent the second and the third invariants of the tensor $\boldsymbol{S}$ :

$$
I_{2}=\frac{1}{2}(1-\boldsymbol{S}: \boldsymbol{S}), \quad I_{3}=\operatorname{det}(\boldsymbol{S})
$$

The numerical values of the parameters, supplied by Grosso et al. (2000b) following the procedure described by Grosso et al. (2000a) are reported in Table I. It is known that the Bingham closure behaves satisfactorily only in weak flows of liquid-crystalline polymers. We also found that, when applied to the modified EFD model, it produces unphysical oscillations of long period before reaching the final steady state for fast shear flows. So, we propose to combine it with the quadratic closure as we have done before for the HL closure. The model obtained will be called MEFD-B if the FENE-LS closure is used for the $\boldsymbol{T}, S_{1}$ and $\boldsymbol{S}_{\mathbf{2}}$ terms and MEFD-BP if the Peterlin closure is used for these. 


\section{Evaluation of the closure approximations}

The Peterlin closure approximation appearing in the evolution equation (12) for the ensemble-averaged segment orientation tensor $\langle\boldsymbol{Q} \boldsymbol{Q}\rangle$ is sufficiently simple that an underlying mean-field-type Fokker-Planck equation may be derived, and an equivalent stochastic equation for the end-to-end vector $\boldsymbol{Q}$ written down, which we show in the first part of this section to be consistent with the fluctuation-dissipation theorem of the first kind (GreenKubo relations for the transport coefficients (Kubo et al. 1985)). A similar analysis for the other closure models being precluded on the grounds of their complexity, we move on in the second part of this section to a comparison of their agreement with the original MEFD model of Fang et al. (2004) in a number of steady and unsteady flows. A valuable discussion of the influence of mean-field approximations on linear response theory and their effect on the interpretation of the Green-Kubo relations may be found in the paper of Hütter and Öttinger (1996).

\subsection{The fluctuation-dissipation theorem and the Green-Kubo relations}

Consider the steady shear flow $\boldsymbol{v}=\left(v_{x}, v_{y}, v_{z}\right)=(\dot{\gamma} y, 0,0)$. Then the evolution equation (24) for $\boldsymbol{A}=\langle\boldsymbol{Q} \boldsymbol{Q}\rangle$ under the Peterlin approximation becomes

$$
\begin{aligned}
\mathbf{0}= & 2 D \boldsymbol{\delta}+\boldsymbol{\kappa} \cdot \boldsymbol{A}+\boldsymbol{A} \cdot \boldsymbol{\kappa}^{T}-\frac{2}{\tau_{s}} \frac{\boldsymbol{A}}{1-\operatorname{tr}(\boldsymbol{A}) / b} \\
& +6\left(\frac{1}{\tau_{s}}-D\right) \frac{\boldsymbol{A}}{\operatorname{tr}(\boldsymbol{A})},
\end{aligned}
$$

with

$$
\boldsymbol{\kappa}=\left(\begin{array}{ccc}
0 & \dot{\gamma} & 0 \\
0 & 0 & 0 \\
0 & 0 & 0
\end{array}\right)
$$

Taking traces of $(55)$ and letting $\dot{\gamma} \longrightarrow 0$ we get the equilibrium value $\operatorname{tr}(\boldsymbol{A})=3 b /(b+3)$. Using this result and the $y y$ and $x y$ components of (55) we may show that the polymeric contribution to the zero shear-rate viscosity $\eta_{p}(0)$ is given by

$$
\begin{aligned}
\eta_{p}(0):=\lim _{\dot{\gamma} \longrightarrow 0} \frac{\tau_{x y}}{\dot{\gamma}} & =\lim _{\dot{\gamma} \longrightarrow 0} \frac{\nu k_{B} T}{\dot{\gamma}} \frac{A_{x y}}{1-\operatorname{tr}(\boldsymbol{A}) / b} \\
& =\frac{\nu k_{B} T}{2 D}\left(\frac{b}{b+3}\right) .
\end{aligned}
$$

The evolution equation (24) for the second moment of the segment configuration $\boldsymbol{A}=\langle\boldsymbol{Q} \boldsymbol{Q}\rangle$ with the FENE$\mathrm{P}$ closure approximation may be derived from an infinite number of different Fokker-Planck equations, amongst them the family of equations

$$
\begin{aligned}
\frac{\partial \psi}{\partial t}= & -\frac{\partial}{\partial \boldsymbol{Q}} \cdot\left[\left(\boldsymbol{\kappa} \cdot \boldsymbol{Q}-\frac{1}{\tau_{s}} \frac{\boldsymbol{Q}}{1-\left\langle Q^{2}\right\rangle / b}\right.\right. \\
& \left.\left.+(\alpha+4 \beta)\left(\frac{1}{\tau_{s}}-D\right) \frac{\boldsymbol{Q}}{\left\langle Q^{2}\right\rangle}\right) \psi\right] \\
& +\frac{\partial}{\partial \boldsymbol{Q}} \frac{\partial}{\partial \boldsymbol{Q}}:\left[\left(D \boldsymbol{\delta}+\beta\left(\frac{1}{\tau_{s}}-D\right) \frac{\boldsymbol{Q} \boldsymbol{Q}}{\left\langle Q^{2}\right\rangle}\right) \psi\right]
\end{aligned}
$$

where we require $\alpha+5 \beta=3$. From the expressions (6) and (8) for $\tau_{\text {eff }}$ and $\tau_{s}$, respectively, we see that under equilibrium conditions $D=1 /\left(3 \tau_{d}\right)$ and $\tau_{s}=$ $\tau_{R 0}$. By choosing $\beta=0$ and considering the equilibrium Fokker-Planck equation we therefore get a drift term which is linear in $\boldsymbol{Q}$, a diffusion term which is constant and a probability density function that is, in consequence, Gaussian. The stochastic differential equation corresponding to (58) in the case $\beta=0$ is

$$
\begin{gathered}
d \boldsymbol{Q}=\left(\boldsymbol{\kappa} \cdot \boldsymbol{Q}-\frac{1}{\tau_{s}} \frac{\boldsymbol{Q}}{1-\left\langle Q^{2}\right\rangle / b}+3\left(\frac{1}{\tau_{s}}-D\right) \frac{\boldsymbol{Q}}{\left\langle Q^{2}\right\rangle}\right) d t \\
+\sqrt{2 D} d \boldsymbol{W}_{t}
\end{gathered}
$$

In equilibrium (59) becomes

$$
d \boldsymbol{Q}=-D\left(\frac{(b+3)}{b}\right) \boldsymbol{Q} d t+\sqrt{2 D} d \boldsymbol{W}_{t},
$$

which has solution

$$
\boldsymbol{Q}(t)=\boldsymbol{\Phi}_{t}\left[\boldsymbol{Q}(0)+\sqrt{2 D} \int_{t^{\prime}=0}^{t} \boldsymbol{\Phi}_{t^{\prime}}^{-1} \cdot d \boldsymbol{W}_{t^{\prime}}\right],
$$

where the propagator $\boldsymbol{\Phi}_{t}$ is given by

$$
\boldsymbol{\Phi}_{t}=\exp \left(-\left(\frac{D(b+3) t}{b}\right) \boldsymbol{\delta}\right) .
$$

According to the Green-Kubo relations (Kubo et al. 1985) the zero shear-rate viscosity should be computable from equilibrium time correlation functions and, in particular, the fluctuation-dissipation theorem of the first kind states that

$$
\begin{aligned}
\eta_{p}(0) & =\frac{1}{\nu k_{B} T} \int_{t=0}^{\infty}\left\langle\tau_{x y}(t) \tau_{x y}(0)\right\rangle_{e q} d t \\
& =\nu k_{B} T\left(\frac{b+3}{b}\right)^{2} \int_{t=0}^{\infty}\left\langle Q_{x}(t) Q_{y}(t) Q_{x}(0) Q_{y}(0)\right\rangle_{e q} d t
\end{aligned}
$$

Since $\boldsymbol{Q}$ is Gaussian with $Q_{x}$ and $Q_{y}$ uncorrelated, we may use the result (see Eq. (2.62) of Öttinger 1996, for example) that

$$
\left\langle Q_{x}(t) Q_{y}(t) Q_{x}(0) Q_{y}(0)\right\rangle=\left\langle Q_{x}(t) Q_{x}(0)\right\rangle\left\langle Q_{y}(t) Q_{y}(0)\right\rangle
$$


where in the present case (see Eq. (3.58) of Öttinger 1996)

$$
\begin{aligned}
\langle\boldsymbol{Q}(t) \boldsymbol{Q}(0)\rangle_{e q} & =\boldsymbol{\Phi}_{t}\langle\boldsymbol{Q}(0) \boldsymbol{Q}(0)\rangle_{e q}, \\
& =\left(\frac{b}{b+3}\right) \exp \left(-\left(\frac{D(b+3) t}{b}\right) \boldsymbol{\delta}\right) .
\end{aligned}
$$

Hence, combining the results (63), (64) and (65) we get

$$
\begin{aligned}
\eta_{p}(0) & =\nu k_{B} T \int_{t=0}^{\infty} \exp \left(-2 D\left(\frac{b+3}{b}\right) t\right) d t, \\
& =\frac{\nu k_{B} T}{2 D}\left(\frac{b}{b+3}\right)
\end{aligned}
$$

which is the same as in Eq. (57).

A similar analysis for the other closure approximations considered in this paper would be difficult, if not impossible, in view of their high degree of complexity.

\subsection{Numerical comparison of the closure approximations}

For the results in this subsection a Brownian dynamics simulation for the modified EFD model was used with between $10^{4}$ and $10^{6}$ configurations and time step sizes $\Delta t$ chosen so that the strain in all flows considered per time step never exceeded 0.02. For small deformation rates the maximum time step size was set at $4 \times 10^{-4}$. For all numerical computations for the closure models a fifthorder Runge-Kutta method with adaptive time step, as described by Press et al. (1989) was used. Further details of our Brownian dynamics method may be gleaned from Fang et al. (2000).

We begin the presentation of our results with a discussion of the extent of agreement with experimental data of predictions of the modified EFD model and its various closure approximations for steady simple shear flow and steady uniaxial extensional flow. We then consider the qualitative behaviour of the models in a doublestep shear strain experiment. In all three flows the choice of rheological parameters is based upon the experimental data of Bhattacharjee et al. $(2002,2003)$ for a $10 \%$ solution of $3.9 \times 10^{6}$ molecular weight polystyrene in diethyl phthalate. The number of entanglements in the solution was calculated by the authors to be $Z=27.4$ and the maximum stretching ratio of the chain contour length to be $\lambda_{\max }=13.6$. The Rouse time was given by the authors as $\tau_{R}=0.282 \mathrm{~s}$ and therefore $\tau_{d}=3 Z \tau_{R}=23.18 \mathrm{~s}$.

3.2.1 Steady shear flow In Fig. 1 (a)-(e) we plot the experimental results of Bhattacharjee et al. (2003) and the predictions of the modified EFD model and its approximations MEFD-P, MEFD-LS, MEFD-Q, MEFDHL and MEFD-B for the shear stress and first normal stress difference in a steady simple shear flow. The reptation times $\tau_{d}$ and elastic moduli $G$ used in the numerical simulations were calculated to give the correct (i.e. experimentally observed) crossover point for the shear stress and first normal stress difference. In this way, $\tau_{d}$ for the modified EFD model has been calculated to be $18 s$, which is smaller than the estimated value $23.18 \mathrm{~s}$ given above. This is because the fast relaxation processes such as contour length fluctuations (CLF) are not taken into account in the model, hence a smaller value of $\tau_{d}$ is necessary to compensate those missing mechanisms. The Rouse time $\tau_{R}$ is chosen to be $0.3 s$, close to the estimated value of Bhattacharjee et al. mentioned above, and $\tau_{R 0}=\tau_{R} / 3=0.1 \mathrm{~s}$. If, like Fang et al. (2004), we apply the relation $\tau_{d} / \tau_{R}=3 Z$ for our present choice of $\tau_{d}$ and $\tau_{R}$, then the resulting value of $Z$ is smaller than that estimated experimentally, which in turn leads to a larger $\lambda_{\max }$ in order to preserve the same total number of Kuhn steps as given by Bhattacharjee et al. (2002, $2003)$. We now believe that it is not proper to recalculate $Z$ from the relation $\tau_{d} / \tau_{R}=3 Z$ by using the value of $\tau_{d}$ obtained from fitting. Therefore, we keep the values for $Z$ and $\lambda_{\max }$ unchanged. For the modified EFD model, and as explained in the paper of Fang et al. (2004), $b$ is taken to be $3 \lambda_{\max }^{2}-5=3 \times(13.6)^{2}-5 \approx 550$.

The same extensibility parameter $b$ and Rouse time $\tau_{R}$ computed for the modified EFD model are also used for each of the closure approximations. $\beta_{1}$ and $\beta_{2}$ in the orientation relaxation time (6) and stretch relaxation time (8), respectively, were both chosen equal to 2 for all the closure models. For the modified EFD model, $\beta_{1}=0.5$ was found to give better results. Since the parameters mentioned above are the same for all the closure models, the proximity of the calculated values of $G$ and $\tau_{d}$ for any closure approximation to the corresponding value for the modified EFD model is a useful quantitative measure of the quality of the agreement, at least in a shear flow.

From the results in Fig. 1 (a)-(e), best overall agreement with the experimental data and the modified EFD results would seem to be achieved by the MEFD-HL closure although, admittedly, the shear stress data is closely matched by the MEFD-P and MEFD-LS models, albeit at the price of $G$ and $\tau_{d}$ parameters that are further from those of the modified EFD model than in the HL and Bingham closures. We note that at higher $\left(>10 s^{-1}\right)$ shear rates the Bingham closure approximation leads to unacceptably high values of both the shear stress and first normal stress difference.

3.2.2 Other flows The same fluid parameters as detailed above for steady shear flow have also been used for simulations of steady uniaxial extensional flow. Agreement with the modified EFD model result was seen to be best using the HL and Bingham closures. However, the modified EFD model, although superior in its agreement with the extensional data to either the coupled 


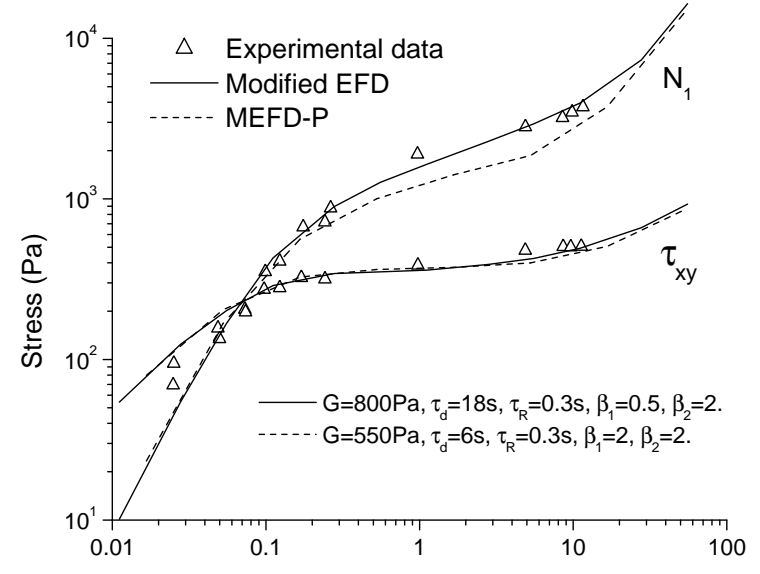

(a)

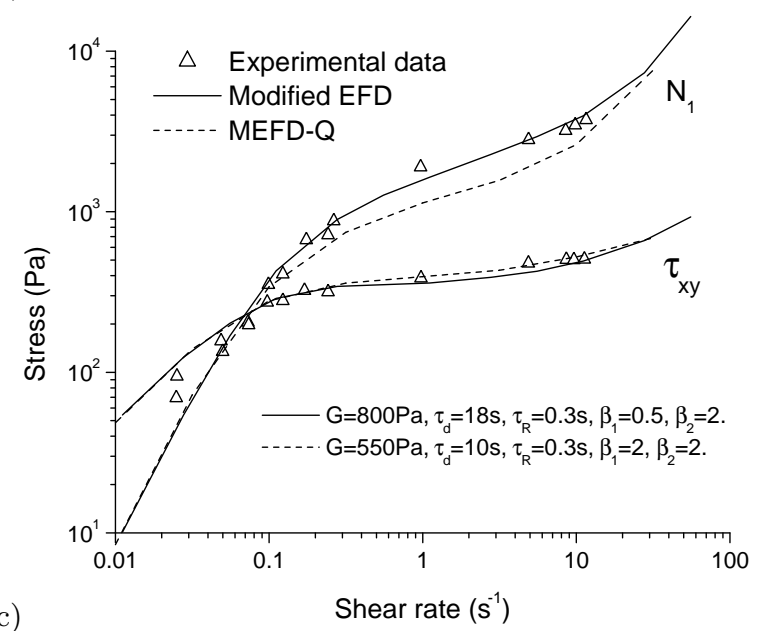

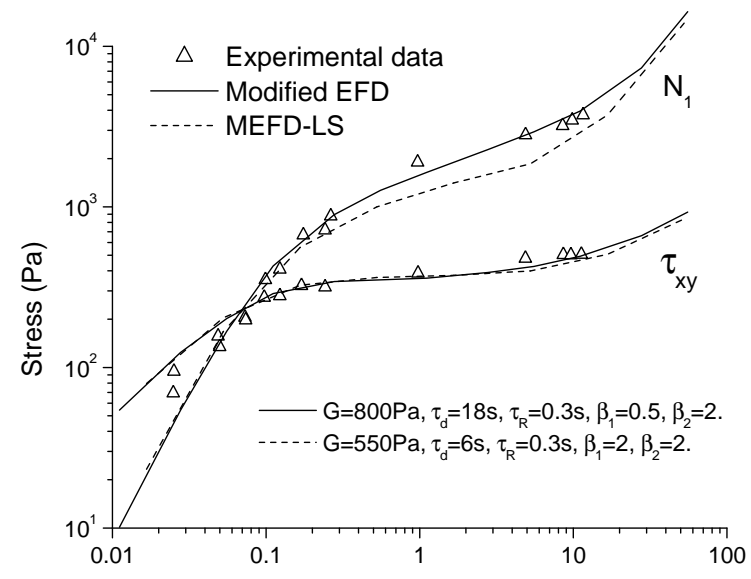

(b)

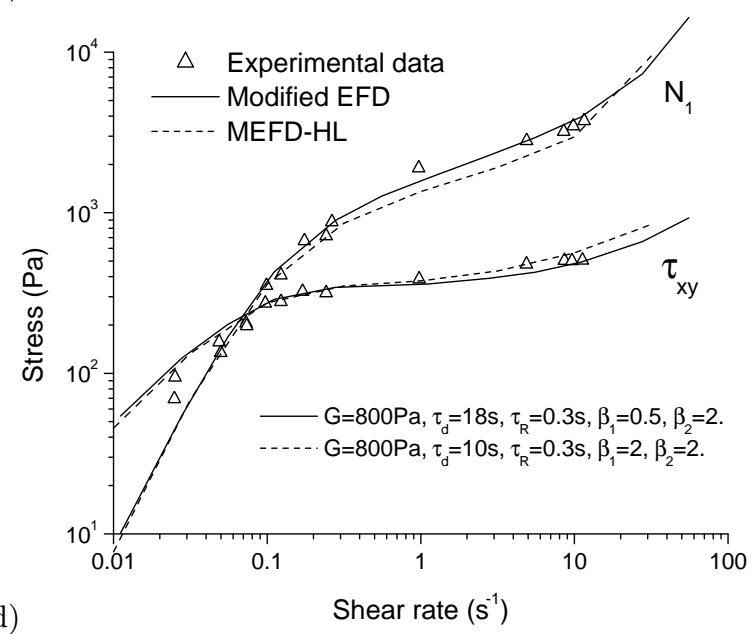

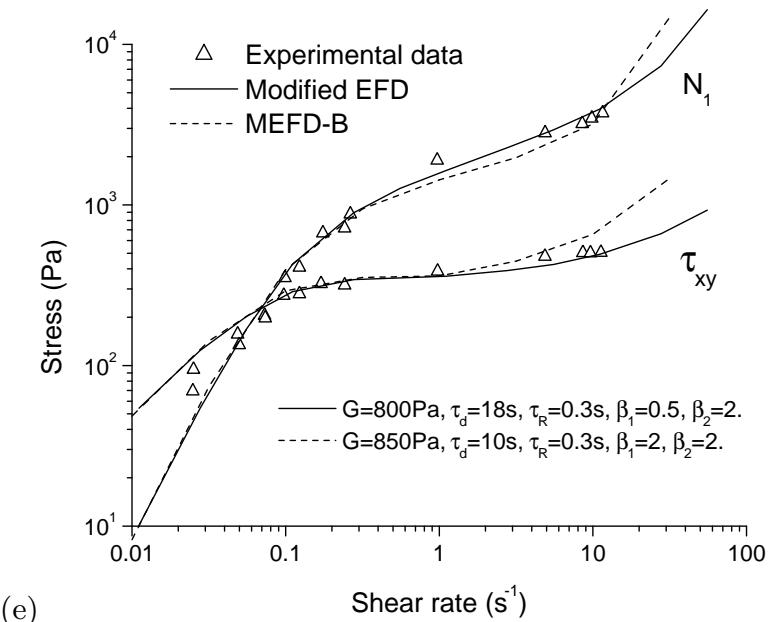

Fig. 1 Steady-state values of shear stress $\tau_{x y}$ and first normal stress difference $N_{1}$ as functions of shear rate $\dot{\gamma}$. Shown are the experimental data of Bhattacharjee et al. (2003), the predictions of the modified EFD model of Fang et al. (2004) and the five closure approximations (a) MEFD-P, (b) MEFD-LS, (c) MEFD-Q, (d) MEFD-HL and (e) MEFD-B. 
DCR-CS model of Marrucci and Ianniruberto (2003) or the original EFD model of Bird and Deaguiar (1983), overpredicts the experimental data for an extension rate $\dot{\varepsilon} \gtrsim 4 s^{-1}$. Improvement on this point is certainly achievable by using a smaller value of $b$ without deterioration of the shear flow results.

In a double-step shear strain experiment a strain of 4 was imposed and then at a time $t_{w}$ (say) later a strain of -2 imposed, giving a total strain of 2 . The same fluid parameters for all models as in the previous two flows described above were used. The expected $\tau_{x y}$ overshoot for $t_{w} / \tau_{d}$ sufficiently small failed to materialize for the MEFD-P, MEFD-LS and MEFD-Q closure approximations. Qualitatively, then, the MEFD-HL and MEFD-B closures return superior results to those of the less sophisticated closures. Better quantitative agreement with the predictions of the modified EFD model was also in evidence.

\subsection{MEFD-HL model. Further results}

Although it has been difficult in the previous section to distinguish between the agreement afforded by the HL and Bingham closures with the modified EFD model predictions for the three flows considered, the slightly better results of the HL closure coupled with its considerably simpler mathematical description make this our closure approximation of choice. In the paragraphs below we study further its properties in some steady and start-up flows.

3.3.1 Start-up shear flow In Fig. 2 (a) and (b) we examine transient growth of the normalized shear stress and first normal stress difference for start-up shear flow, as predicted by the modified EFD model and the HL closure. The experimental data (denoted by symbols in the two figures) are taken from the thesis of Kahvand (1995). The test fluid is a solution in tricresyl phosphate of nearly monodisperse polystyrene with a molecular weight $M_{w}$ of $1.9 \times 10^{6}$ (polydispersity index of 1.2 ) and a polymer density of $0.135 \mathrm{~g} / \mathrm{cm}^{3}$. The reptation time $\tau_{d}$ and the average number of entanglements $(Z)$ of the fluid are estimated to be $15 \mathrm{~s}$ and 10 , respectively. In Fang et al. (2000) $Z$ was taken equal to 7 with 441 Kuhn steps per entanglement. Hence, we here estimate the number of Kuhn steps per entanglement to be $7 \times 441 / 10=308.7$. The maximum chain stretching ratio of the fluid is then $\lambda_{\max }=\sqrt{308.7} \approx 17.6$ so that for both the modified EFD model and its closure approximation $b$ should be chosen to be $3 \lambda_{\max }^{2}-5 \approx 924$ (see Fang et al. 2004 for details). The modulus $G$ and the reptation time for the modified EFD model were determined in the usual way from the steady shear data of Kahvand (1995) to be $1500 \mathrm{~Pa}$ and 13.5s, respectively. The Rouse time is $\tau_{R}=15 / 30=0.5 \mathrm{~s}$, which is not far from the estimated value $(0.75 \mathrm{~s})$ of Venerus and Kahvand (1994). The values for the HL closure of $G$ and $\tau_{d}$

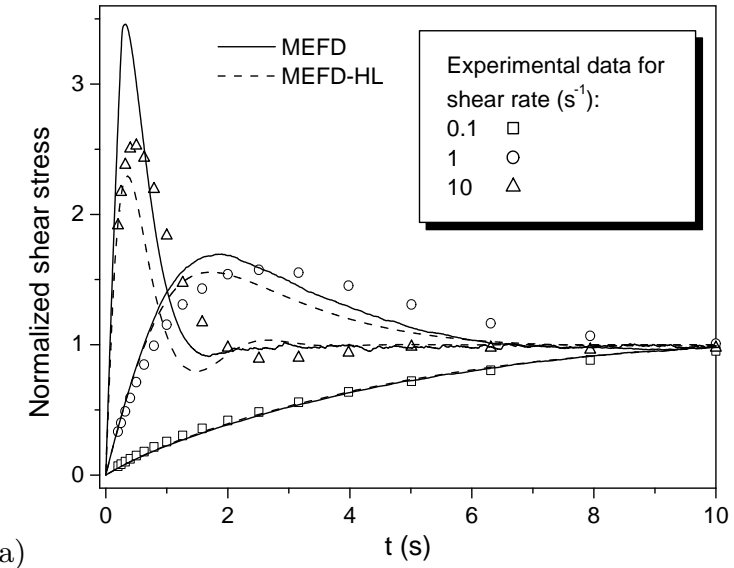

(a)

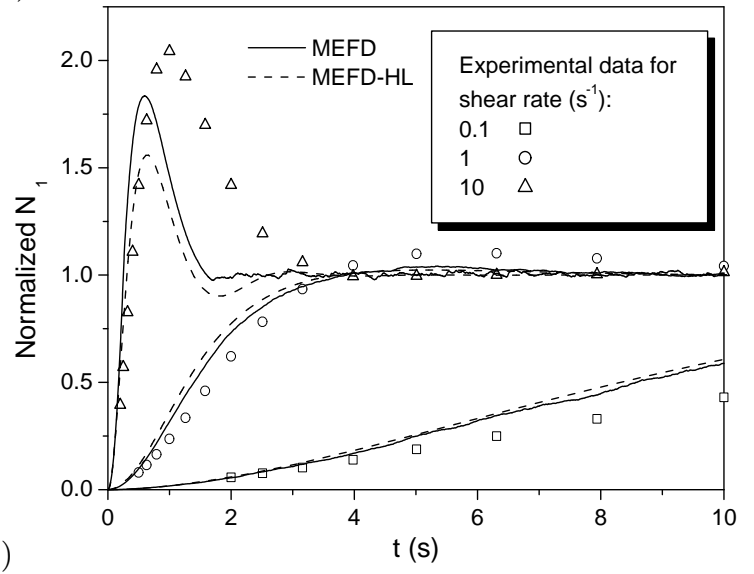

Fig. 2 Transient growth of (a) normalized shear stress and (b) first normal stress difference as a function of time under start-up shear flow at several shear rates observed experimentally by Khavand (1995) and predicted by the modified EFD model of Fang et al. (2004) and the closure approximation MEFD-HL.

were calculated to be $1500 \mathrm{~Pa}$ and $9 \mathrm{~s}$, and the same value $0.5 \mathrm{~s}$ was used for $\tau_{R}$.

From Fig. 2 (a) and (b) it may be seen that the modified EFD model overpredicts the $\tau_{x y}$ overshoot at the highest shear rate $\dot{\gamma}=10 \mathrm{~s}^{-1}$ but underpredicts that of $N_{1}$. Behaviour at the lowest shear rate for the modified EFD model and the HL closure is comparable. The HL closure underpredicts the peak values of both the shear stress and first normal stress difference at $\dot{\gamma}=10 \mathrm{~s}^{-1}$ but in so doing returns better agreement with the experimental data for small times. As observed by Fang et al. (2004), we see from Fig. 2 (a) and (b) that a physical overshoot in $\tau_{x y}$ occurs even at $\dot{\gamma}=1$ and thus we concur with the observation made in Fang et al. (2000) that an overshoot in $N_{1}$ occurs at a higher shear rate than that in $\tau_{x y}$. At $\dot{\gamma}=10 \mathrm{~s}^{-1}$ the peak value of $N_{1}$ is attained later than that of $\tau_{x y}$.

3.3.2 Computation of $\langle\boldsymbol{u} \boldsymbol{u}\rangle$ in the constraint release rate $k$. In Fig. 3 we demonstrate the curious and striking 
difference of behaviour of the HL closure when $\boldsymbol{S}$ from Eq. (42) and $\boldsymbol{A} / \operatorname{tr}(\boldsymbol{A})$ are used in the calculation of the constraint release rate $k$ in (7) for a start-up shear flow at a dimensionless shear rate $\dot{\gamma} \tau_{d}=1000$. Also shown is the prediction of the normalized shear stress using the modified EFD model of Fang et al. (2004). All relevant parameters for both the modified EFD model and the closure models are set to $Z=20, \beta_{1}=0.5$, and $\beta_{2}=2$. It may be seen that a huge (and unphysical) overshoot in the normalized shear stress is possible when $\boldsymbol{S}$ in the HL closure is used to calculate $k$, and that the solution persistently oscillates thereafter. In shear flow, $k$ is only

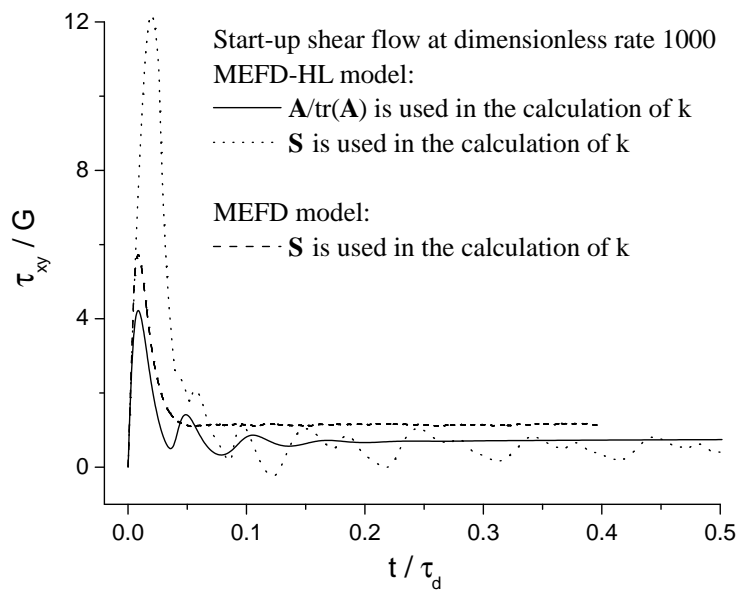

Fig. 3 Behavior of normalized shear stress as a function of time under start-up shear flow at a dimensionless shear rate $\dot{\gamma} \tau_{d}$ of 1000 . Shown are the results of using $\boldsymbol{S}$ from Eq. (42) and $\boldsymbol{A} / \operatorname{tr}(\boldsymbol{A})$ in the calculation of the constraint release rate $k$ in (7) for the MEFD-HL model and the results from the modified EFD model.

affected by the $(x, y)$-component of $\boldsymbol{S}$ or $\boldsymbol{A} / \operatorname{tr}(\boldsymbol{A})$ and we therefore show the behavior of these components in Fig. 4. Comparing $A_{x y} / \operatorname{tr}(\boldsymbol{A})$ and $S_{x y}$ predicted by the MEFD-HL model using $\boldsymbol{A} / \operatorname{tr}(\boldsymbol{A})$ in the calculation of $k$, we see that the former is in good agreement with $S_{x y}$ from the modified EFD model, while the latter oscillates unrealistically. Not shown are $A_{x y} / \operatorname{tr}(\boldsymbol{A})$ and $S_{x y}$ predicted by the MEFD-HL model using $\boldsymbol{S}$ in the calculation of $k$, for which $A_{x y} / \operatorname{tr}(\boldsymbol{A})$ behaves similarly to the previous case, while $S_{x y}$ manifests huge oscillations and is far from realistic. The above observations explain why $\boldsymbol{A} / \operatorname{tr}(\boldsymbol{A})$ is superior to $\boldsymbol{S}$ for the evaluation of the constraint release rate $k$.

3.3.3 Use of the Peterlin approximation (46) In Fig. 5 we show the result of superimposing on Fig. 1 (d) the predictions for the shear stress and first normal stress difference obtained for the same steady shear flow using the MEFD-HLP model (that is the HL closure (51) for $\boldsymbol{R}$ and the Peterlin approximation (23) for $\boldsymbol{T}$ and (46) for $S_{1}$ and $\boldsymbol{S}_{2}$, respectively). An advantage in favour of

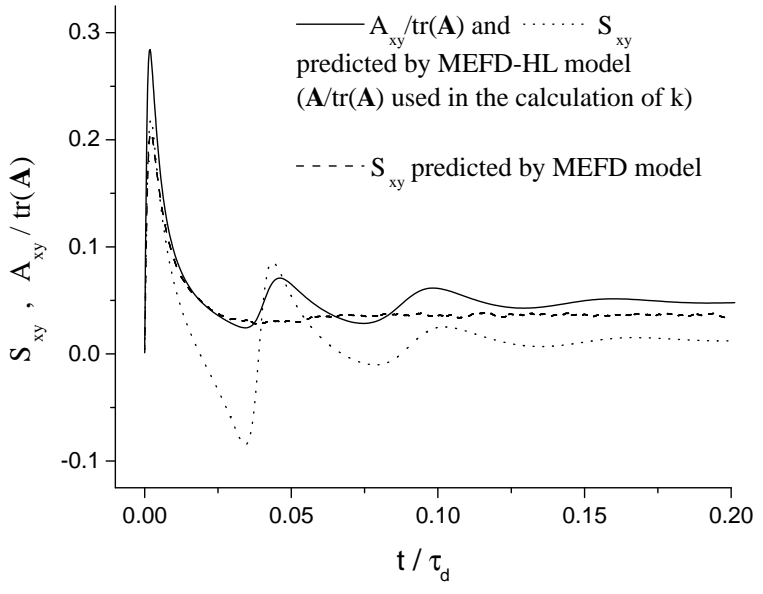

Fig. 4 Behavior of the $(x, y)$-component of $\boldsymbol{S}$ and $\boldsymbol{A} / \operatorname{tr}(\boldsymbol{A})$ as a function of time under start-up shear flow at a dimensionless shear rate $\dot{\gamma} \tau_{d}$ of 1000 . Shown are the results of using $\boldsymbol{A} / \operatorname{tr}(\boldsymbol{A})$ in the calculation of the constraint release rate $k$ in (7) for the MEFD-HL model and the results from the modified EFD model.

the MEFD-HL model, i.e., with the LS evaluation of $\boldsymbol{T}$, $S_{1}$, and $\boldsymbol{S}_{2}$, is discernible by looking at the value of $\tau_{d}$ calculated, which is closer to that of the modified EFD model than in the case of the Peterlin approximation.

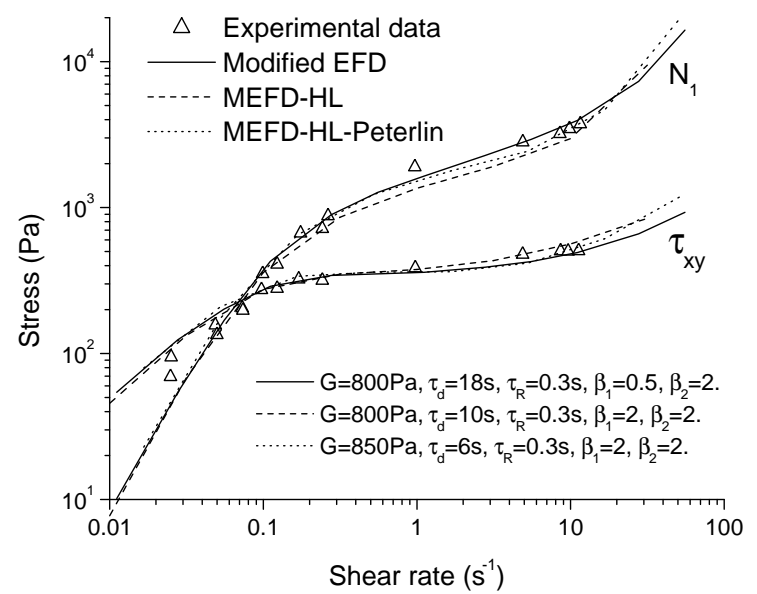

Fig. 5 Steady-state values of shear stress $\tau_{x y}$ and first normal stress difference $N_{1}$ as functions of shear rate $\dot{\gamma}$. Shown are the experimental data of Bhattacharjee et al. (2003), the predictions of the modified EFD model and the Peterlin and LS versions of the MEFD-HL closure approximation.

3.3.4 Start-up extensional flow Finally, we present results of simulations using the modified EFD model and the MEFD-HL closure approximation for start-up uniaxial extensional flow. The numerical results in Figs. 6 (a) and (b) are compared with the experimental data of 


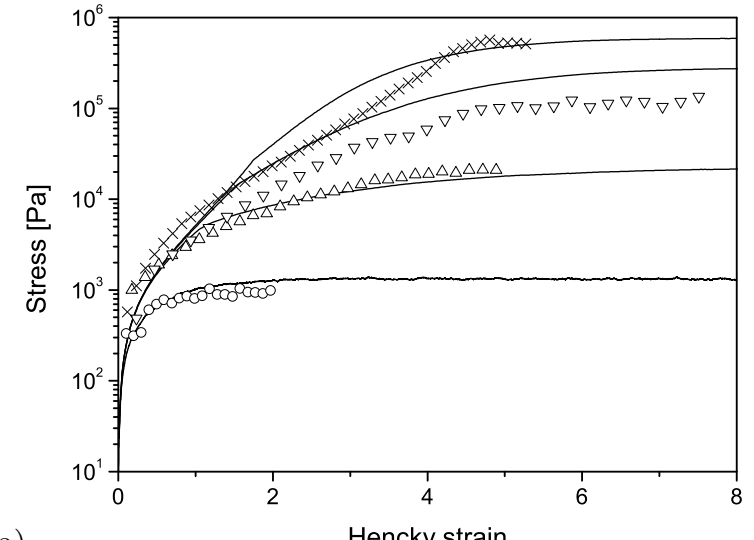

(a)

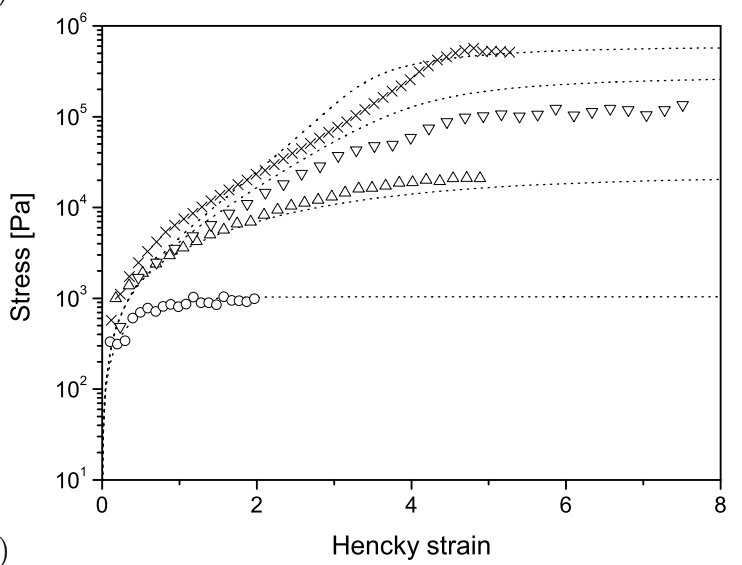

(b)

Fig. 6 Transient extensional stress response in uniaxial extensional flow plotted against Hencky strain. Shown are the extensional data of Bhattacharjee et al. (2003) at strain rates $\dot{\varepsilon}=0.1 \mathrm{~s}^{-1}(\circ), \dot{\varepsilon}=3.5 \mathrm{~s}^{-1}(\triangle), \dot{\varepsilon}=7.3 \mathrm{~s}^{-1}(\nabla)$ and $\dot{\varepsilon}=11.7 \mathrm{~s}^{-1}(\times)$. (a) Comparison with predictions of the modified EFD model of Fang et al. (2004). (b) Comparison with predictions of the closure approximation MEFD-HL.

Bhattacharjee et al. (2003) for a $10 \%$ solution of $3.9 \times 10^{6}$ molecular weight polystyrene diethyl phosphate. Material parameters were taken to be the same as those detailed in Fig. 1(d) except that a smaller value of $b=295$ (corresponding to $\lambda_{\max }=10$ ) was selected in order to give better fitting of data at high extensional rates. The numerical results from both models are in close agreement with the experimental data over the chosen range of strain rates and are hardly distinguishable from each other. Arguably, agreement with the experimental data is superior to that obtained with the DCR-CS model of Ianniruberto and Marrucci (2001), the use of which as observed by Bhattacharjee et al. (2003) led, with the exception of the data at the lowest strain rate, to consistent under-prediction of the experimental data (see Fig. 6(a) of Bhattacharjee et al. 2003).

\section{Conclusions}

In this paper we have compared the performance of combinations and adaptations of five different closure approximations in their capacity to faithfully reproduce the predictions of the modified encapsulated FENE dumbbell model of Fang et al. (2004) for a number of shear and extensional flows. Comparison has also been made with the experimental data of Kahvand (1995) and Bhattacharjee et al. (2002, 2003). In the case of the HinchLeal and Bingham closures (Hinch and Leal 1976; Chaubal and Leal 1998) a combination with the quadratic closure of Doi (1981) was found to be necessary for fast flows. The Hinch-Leal closure approximation, modified in this way, was found to outperform the other closures and its mathematical description is considerably simpler than that of the Bingham closure. We therefore recommend the use of the closure model, MEFD-HL, which includes the time evolution equations (12), (27), and (42) for the three state variables $\boldsymbol{A}, B$, and $\boldsymbol{S}$, respectively, the modified Hinch-Leal closure (51) for $\boldsymbol{R}$ and the LS approximations (29) and (47) to $\boldsymbol{T}, S_{1}$ and $\boldsymbol{S}_{2}$. In the case of the Peterlin approximation (Peterlin 1966) a mean-fieldtype Fokker-Planck equation underlying the evolution equation for an equilibrium averaged polymer segment orientation tensor is shown to be consistent with the fluctuation-dissipation theorem (Kubo et al. 1985).

Acknowledgements The authors would like to thank Massimiliano Grosso for his help on the application of the Bingham closure and pointing out some typing errors in the paper by Grosso et al. (2000a). The authors would also like to thank Markus Hütter for useful discussions on the subject of the fluctuation-dissipation theorem. The insights provided by the referees are acknowledged with gratitude.

\section{References}

1. Advani SG, Tucker CL (1987) The use of tensors to describe and predict fiber orientation in short fiber composites. J Rheol 31: 751-784.

2. Bhattacharjee PK, Nguyen DA, McKinley GH, Sridhar T (2003) Extensional stress growth and stress relaxation in entangled polymer solutions. J Rheol 47: 269-290.

3. Bhattacharjee PK, Oberhauser JP, McKinley GH, Leal LG, Sridhar T (2002) Extensional rheometry of entangled solutions. Macromolecules 35: 10131-10148.

4. Bird RB, Deaguiar JR (1983) An encapsulated dumbbell model for concentrated polymer solutions and melts I. Theoretical developments and constitutive equation. J Non-Newtonian Fluid Mech 13: 149-160.

5. Bird RB, Curtiss CF, Armstrong RC, Hassager O (1987) Dynamics of Polymeric Liquids, vol.2, Kinetic theory, 2nd edn. Wiley-Interscience, New York.

6. Chaubal CV (1997) Ph.D. thesis, University of California, Santa Barbara.

7. Chaubal CV, Leal LG (1998) A closure approximation for liquid-crystalline models based on parametric density estimation. J Rheol 42: 177-201. 
8. Chauvière C, Lozinski L (2004) Simulation of dilute polymer solutions using a Fokker-Planck equation. Computers and Fluids 33:: 687-696.

9. Cintra JS, Tucker CL (1995) Orthotropic closure approximations for flow-induced fiber orientation. J Rheol 39: 1095-1122.

10. des Cloizeaux J (1988) Double reptation vs simple reptation in polymer melts. Europhys Lett 5: 437-442.

11. Doi M (1981) Molecular dynamics and rheological properties of concentrated solutions of rodlike polymers in isotropic and liquid crystalline phases. J Polym Sci, Polym Phys Ed 19: 229-243.

12. Doi M, Edwards SF (1978a) Dynamics of concentrated polymer systems: Brownian motion in the equilibrium state. J Chem Soc Faraday Trans 74: 1789-1801.

13. Doi M, Edwards SF (1978b) Dynamics of concentrated polymer systems: Molecular motion under flow. J Chem Soc Faraday Trans 74: 1802-1817.

14. Doi M, Edwards SF (1978c) Dynamics of concentrated polymer systems: The constitutive equation. J Chem Soc Faraday Trans 74: 1818-1832.

15. Fang J, Kröger M, Öttinger HC (2000) A thermodynamically admissible reptation model for fast flows of entangled polymers. II. Model predictions for shear and extensional flows. J Rheol 44: 1293-1317.

16. Fang J, Lozinski A, Owens RG (2004) Towards more realistic kinetic models for concentrated solutions and melts. J Non-Newtonian Fluid Mech 122: 79-90.

17. Feng J, Chaubal CV, Leal LG (1998) Closure approximations for the Doi theory: Which to use in simulating complex flows of liquid-crystalline polymers? J Rheol 42: 1095-1119.

18. Grosso M, Maffettone PL, Dupret F (2000a) A closure approximation for nematic liquid crystals based on the canonical distribution subspace theory. Rheol Acta 39: 301-310.

19. Grosso M, Maffetone PL, Halin P, Keunings R, Legat V (2000b) Flow of nematic polymers in eccentric cylinder geometry: influence of closure approximations. J NonNewtonian Fluid Mech 94: 119-134.

20. Hand GL (1962) A theory of anisotropic fluids. J Fluid Mech 13:33-46.

21. Hinch EJ, Leal LG (1975) Constitutive equations in suspension mechanics. Part 1. General formulation. J Fluid Mech 71: 481-495.

22. Hinch EJ, Leal LG (1976) Constitutive equations in suspension mechanics. Part 2. Approximate forms for a suspension of rigid particles affected by Brownian rotations. J Fluid Mech 76: 187-208.

23. Hütter M, Öttinger HC (1996) Modification of linear response theory for mean-field approximations. Phys. Rev. E 54: 2526-2530.

24. Ianniruberto G, Marrucci G (1996) On compatibility of the Cox-Merz rule with the model of Doi and Edwards. J Non-Newtonian Fluid Mech 65: 241-246.

25. Ianniruberto G, Marrucci G (2000) Convective orientational renewal in entangled polymers. J Non-Newtonian Fluid Mech 95: 363-374.

26. Ianniruberto G, Marrucci G (2001) A simple constitutive equation for entangled polymers with chain stretch. J Rheol 45: 1305-1318.
27. Ianniruberto G, Marrucci G (2002) A multi-mode CCR model for entangled polymers with chain stretch. J NonNewtonian Fluid Mech 102: 383-395.

28. Kahvand H (1995) PhD Thesis: Strain Coupling Effects in Polymer Rheology, Illinois Institute of Technology.

29. Kubo R, Toda M, Hashitsume N (1985) Statistical Physics II: Non-equilibrium Statistical Mechanics, 2nd ed. Springer, New York.

30. Lielens G, Keunings R, Legat V (1999) The FENE-L and FENE-LS closure approximations to the kinetic theory of finitely extensible dumbbells. J Non-Newtonian Fluid Mech 87: 179-196.

31. Lozinski A, Chauvière (2003) A fast solver for FokkerPlanck equation applied to viscoelastic flow calculations: 2D FENE model. J Comp Phys 189: 607-625.

32. Lozinski A, Chauvière C, Fang J, Owens RG (2003) Fokker-Planck simulations of fast flows of melts and concentrated polymer solutions in complex geometries. J Rheol 47: 535-561.

33. Lozinski A, Owens RG, Fang J (2004) A Fokker-Planckbased numerical method for modelling non-homogeneous flows of dilute polymeric solutions. J Non-Newtonian Fluid Mech 122: 273-286.

34. Maffettone PL (1992) A constitutive equation for monodomains of nematic polymers. J Non-Newtonian Fluid Mech 45: 339-354.

35. Marrucci G (1996) Dynamics of entanglements: A nonlinear model consistent with the Cox-Merz rule. J NonNewtonian Fluid Mech 62: 279-289.

36. Marrucci G, Grizzuti N (1988) Fast flows of concentrated polymers - predictions of the tube model on chain stretching. Gazz Chim Ital 118: 179-185.

37. Marrucci G, Ianniruberto G (2003) Flow-induced orientation and stretching of entangled polymers, Phil Trans R Soc Lond A 361: 677-687.

38. Mead DW, Larson RG, Doi M (1998) A molecular theory for fast flows of entangled polymers. Macromolecules 31 : 7895-7914.

39. Mead DW, Leal LG (1995) The reptation model with segmental stretch I. Basic equations and general properties. Rheol Acta 34: 339-359.

40. Mead DW, Yavich D, Leal LG (1995) The reptation model with segmental stretch II. Steady flow properties. Rheol Acta 34: 360-383.

41. Öttinger HC (1994) Modified reptation model. Phys Rev E 50: 4891-4895.

42. Öttinger HC (1996) Stochastic Processes in Polymeric Fluids: Tools and Examples for Developing Simulation Algorithms, Springer, Berlin.

43. Öttinger HC (1999) A thermodynamically admissible reptation model for fast flows of entangled polymers. J Rheol 43: 1461-1493.

44. Pearson DS, Herbolzheimer EA, Marrucci G, Grizzuti N (1991) Transient behavior of entangled polymers at high shear rates. J Polym Sci Polym Phys Ed 29: 1589-1597.

45. Peterlin A (1966) Hydrodynamics of macromolecules in a velocity field with longitudinal gradient. J Polym Sci B 4: 287-291.

46. Press WH, Flannery BP, Teukolsky SA, Vetterling WT (1989) Numerical Recipes. The Art of Scientific Computing. Cambridge University Press, Cambridge, UK. 
47. Schieber JD (1992) Do internal viscosity models satisfy the fluctuation-dissipation theorem? J. Non-Newtonian Fluid Mech 45: 47-61.

48. Schieber JD, Öttinger HC (1988) The effects of bead inertia on the Rouse model, J. Chem. Phys. 89:6972-6981.

49. Tsenoglou C (1987) Viscoelasticity of binary homopolymer blends. ACS Polym. Preprints 28: 185-186.

50. van Meerveld J. (2002) Private communication.

51. Venerus DC, Kahvand H (1994) Doi-Edwards theory evaluation in double-step strain flows, J Polym Sci, Part B: Polym Phys 32: 1531-1542.

52. Zmievsky VB, Karlin IV, Deville M (2000) The universal limit in dynamics of dilute polymeric solutions, Physica A 275: 152-177. 